\title{
Selective Impairment of the "Central Executive" Component of Working Memory: A Single Case Study
}

\author{
M. Van der Linden \\ Unité de Neuropsychologie Cognitive, Université de Louvain, Belgium \\ F. Coyette \\ Cliniques Universitaires Saint-Luc, Université de Louvain, Belgium \\ $X$. Seron \\ Unité de Neuropsychologie Cognitive, Université de Louvain, Belgium
}

We report the case of a head-injured patient (AM) with a specific short-term memory impairment. He did not show any deficit in long-term memory and memored no sign of either intellectual or frontal dysfunction. His spans for verbal and nonverbal material were reduced and he showed a lack of recency verfal and nonverbal material were recall for visually presented words. We observed word length and phonological similarity effects as well as the abolition with articulatory and phonological similarity effects as werd length effect and of the visual phonological similarity effect, indicating that both the phonological store and the articulatory rehearsal mechanism were functioning. Furthermore, our investigations did not evidence any structural defect in either the control and planning functions of the central executive, or in its storage functions. The deficit AM presented on a verbal and nonverbal Brown-Peterson task, especially when the distractor tasks were more demanding, suggests that AM's central executive disposed of fewer processing resources. This resource reduction seemed to affect only short-term storage but not processing, and was interpreted as the consequence of strategic adaptation by the patient. Finally, the resource reduction did not cause interference on those divided attention tasks not requiring storage. As such, these results call for a more accurate specification of the allocation of resources in dual tasks.

Address correspondence and reprint requests to Martial Van der Linden, Unité de Neuropsychologie Cognitive, Faculté de Psychologie, Voie du Roman Pays, Louvain-LaNeuve, Belgium.

An earlier version of this paper was presented at the Société de Neuropsychologie de Langue Française meeting in Paris, December 1988.

Martial Van der Linden is presently supported as a Research Associate by the National Fund for Scientific Research (Belgium).

(C) 1992 Lawrence Erlbaum Associates Limited 


\section{INTRODUCTION}

Confronted with the numerous problems inherent in the short-term memory (S.T.M.) conception in Atkinson and Shiffrin's (1968) model, Baddeley and Hitch (1974) decided to abandon the idea of a unitary shortterm storage system and replaced this view with a model that assumes a multi-component working memory concept. This concept refers to a general and central system that is responsible for the processing and temporary storage of information in the realisation of varying cognitive tasks.

The working memory model comprises a modality-free controlling central excutive of limited capacity that is aided by a number of subsidiary slave systems, which are responsible for temporary maintenance of information. Two such systems have been explored in more detail: the articulatory loop and the visuospatial sketchpad (see Baddeley, 1986, for an extensive presentation of the working memory model).

The visuospatial sketchpad system is assumed to be concerned with the setting up and the maintenance of visuospatial images. The articulatory loop system is specialised for processing verbal material and it is composed of two subsystems: a phonological store and an articulatory rehearsal process. The phonological store receives directly and obligatorily any information auditorily presented and stores it in terms of a sound-based code. Although material in this store is subject to decay and interference, it can be maintained and reinforced through the articulatory rehearsal mechanism. The phonological store is also able to receive visually presented items but these must first have been converted into an articulatory form before gaining access to the store. These items are conveyed to the store by the articulatory rehearsal process.

The core of the working memory model is the central executive. It is assumed to play a role in the selection, the planning, and the control of the various processes used in short-term storage and more general processing tasks. Following Morris and Jones (1990), it is especially involved in the co-ordination of working memory updating in real time. This central processor also contributes to the regulation of the slave systems and to the integration of information from these and from longterm memory. Furthermore, according to Vallar and Baddeley (1982), it also has a specific storage function, namely, responsibility for operating maintenance rehearsal. Finally, an important characteristic of this system is that it has a limited processing capacity to be divided between its different control and storage functions. Baddeley (1986) has suggested that the S.A.S. component of the attentional control of action model proposed by Norman and Shallice (1986) might be an adequate approximation of the central executive system. Following Norman and Shallice, some action schemata (routine actions) can run themselves off automatically, whereas, er actions require deliberate attentional resources. These authors assume the existence of two attentional control processes: the contention scheduling mechanism, which should be involved in the selection between conflicting routine actions, and the supervisory attentional system (S.A.S.), which should intervene when the selection of routine actions is not sufficient (for instance, in tasks that require planning or decision making, or when coping with novel or dangerous situations). According to Shallice $(1982 ; 1988)$, a dysfunctioning of the S.A.S. system could plausibly account for the cognitive deficits following frontal-lobe lesions.

The performance of particular patients with a specific deficit of S.T.M. has been claimed to provide evidence for the existence of the different components of the working memory model. Thus, some patients have presented a deficit of auditory verbal S.T.M. that was imputed either to a selective impairment of the phonological store (Vallar \& Baddeley, 1984) or to a disturbance of the articulatory rehearsal process (Belleville, Peretz, \& Arguin, Note 2). Furthermore, a failure of the central executive was assumed to be the cause of the S.T.M. defect observed in Alzheimer's disease (A.D.) (Baddeley et al., 1986; Morris, 1986; Morris \& Baddeley, 1988). This central executive dysfunction in A.D. patients was investigated by means of dual-task procedures in which the patients must divide their attention between ongoing processing and short-term storage (Baddeley et al., 1986; Morris, 1986). Several authors have also suggested that the elderly have difficulty on tasks that make demands on the central executive (Light \& Anderson, 1985; Wright, 1981). Recent studies support the hypothesis that older people have a smaller storage capacity (Foos, 1989), whereas others favour a processing deficit (Morris, Gick, \& Craik, 1988). Finally, rejoining Shallice's proposal, Baddeley (1986) has argued that the central executive is involved in the "dysexecutive" frontal lobe syndrome.

Following Baddeley (1986), the central executive is certainly not a unitary system and, as a consequence, it could be damaged in different ways. Thus, he distinguishes between at least two different alterations to ways. Thus, he distinguishes between at least two different alterations deficit of the control and planning component (flexibility). In this regard, frontal patients would show a deficit of the control processes, the elderly a reduction in the processing resources, and the Alzheimer patients a problem affecting both aspects.

Baddeley (1986; Baddeley et al., 1986) admits that little is known about the workings of the central executive. Yet he suggests applying this model, despite its limitations, to the analysis of neuropsychological problems despite its limitations, to the analysis of neuropsychological problems ponents of the central executive system. We think this also holds for the neuropsychological study that follows. The study involves a head-injured neuropsychological study that follows. The study involves a head-injured 
to an impairment of the "central executive" system and particularly to a reduction in the central processing capacity. AM showed no sign of intellectual deterioration, no frontal dysfunction, and no attentional deficits We shall suggest that only the processes implicated in short-term storage were affected by AM's resources reduction. The results will be discussed with regard to the internal structure and functions of the central executive.

\section{CASE HISTORY}

AM is a 29-year-old right-handed man who was employed as a geometrician. In August 1987 he sustained a severe head injury in a traffic accident. We have no detailed information concerning the immediate posttraumatic events, except that the patient was comatose, presented multiple fractures, and showed no signs of lateralisation upon clinical examination or at the E.E.G. recording. The patient had been placed under barbiturate coma for 3 weeks.

AM came to the Neurological Unit of the St Luc Hospital at Brussels in January 1988, four months after the accident. At that time, the patient's complaints were mainly memory deficits, hypersomnia, cephalalgia, and irritability. At the neurological examination, the tendon reffexes were increased and plantar reflexes were in flexion. A diminution of the superficial sensitivity of the left inferior limb was observed. There were also dysaesthesia after thermoalgesic, pinprick, and proprioceptive stimulations. Magnetic resonance imaging (M.R.I.) showed restricted left lowintensity fronto-basal lesions, a left hyper-intensity white matter lesion in the left frontal periventricular area, a cerebellar and a moderate bilateral frontal atrophy.

A short neuropsychological examination administered in February 1988 was normal for language, intellectual, and attentional functions. More specifically, the patient was alert and well oriented in time and space. Oral language was fluent, without lexical or syntactical deficits, and comprehension was essentially normal. Reading and writing evidenced no difficulties. Mental and written simple calculation were normal and there were no signs of apraxia and agnosia. Long-term memory assessment indicated normal results in a verbal and a nonverbal memory task. On a 15-wordlist learning task (Selective Reminding Task, Buschke \& Fuld, 1974), AM recalled an average of 12.6 words per trial (controls: $12.8, \mathrm{~s} d=0.69$ ) and his C.L.T.R.L.T.R (consistent long-term retrievallong After al/long-term retrieval) score was of $79.3 \%$ (controls: $85 \%$, s.d. $=11$ ). After a 30 -min. delay, the patient recalled 15 words, achieving the maximum score. On the Complex Figure Test (Rey, 1959), AM scored 31 on the memory reproduction task: His performance was at the 100 th percentile. The only weak memory performances were a forward digit span of 4 , a backward digit span of $3 !$ echsler,
1955 ) and a visuospatial span of 4 (Corsi block-tapping procedure, Milner, 1971).

In May 1988, AM was directed towards the Neuropsychological Revalidation Unit in order to undertake a re-education. AM complained essentially about memory problems in his professional activities. In particular, he mentioned being unable to read scientific papers owing to the fact that he would forget the information he had just read and he was therefore frequently obliged to reread parts of the paper. Moreover, he could not attend a technical conversation with clients because he could not understand and memorise simultaneously what the clients said. These difficulties appeared to be only for technical information, which requires effortful processing. Finally, as a geometrician, he had to take many measures and to memorise these measures before transcribing them into a notebook; before his accident, he was able to maintain 8-digit numbers in memory, a performance he was unable to carry out after his accident.

A detailed neuropsychological examination was carried out between May and September 1988 . The characteristics of the memory complaints and the existence of an impaired span with normal performance for the and the existence of an impaired span with normal performance for the AM's working memory efficiency more deeply.

\section{NEUROPSYCHOLOGICAL EXAMINATION}

\section{Methodological Remarks}

For all the tasks we presented to AM, performance was compared either to already existing norms or, if such norms did not exist, to the scores obtained by a group of 10 normal adult subjects of the same educational level and age (mean: 25.5 ; range: $22-34$ ).

\section{Attentional Functions}

A three-component attentional battery of tests implemented on an Apple Ale microcomputer and elaborated according to Posner's conception of attentional processing was administered (Posner \& Boies, 1971).

Alertness. The subject had to react to a centrally located stimulus preceded by an advertising signal (the 8 signal-stimulus intervals varied from $0.1-1.5 \mathrm{sec}$. in steps of $0.2 \mathrm{sec}$.). There were 3 conditions: visual, auditory, and visuo-auditory signal. There were 40 signal-stimulus pairs by condition, 5 by each interval duration. AM's results were normal for each of the 3 conditions: he obtained a median reaction time of $201 \mathrm{msec}$. (controls: $256 \mathrm{msec}$.) in the visual indition, $230 \mathrm{msec}$. (controls: $244 \mathrm{msec}$.) in the 
auditory condition, and $232 \mathrm{msec}$. in the visuo-auditory condition (controls: $246 \mathrm{msec}$.).

Selectivity. A signal was presented in the centre of the screen for $300 \mathrm{msec}$, then it was replaced by an arrow pointing to the left or the right. After a delay of 0.5 or 1 second, the arrow disappeared and a red or blue square appeared on the left or right side of the screen. The subject had to select 1 of 2 coloured buttons according to the colour of the stimulus. In $80 \%$ of the trials, the stimulus was presented in the cued part of the screen (congruent signal-stimulus essays); in $20 \%$, the stimulus was not congruent (non-congruent signal-stimulus essays). For all task conditions, AM scored a few above the normal range. He obtained a mean reaction time of $429 \mathrm{msec}$. (controls: $480 \mathrm{msec}$, s.d.: 81 ). His rate of error was negligible and in the normal range.

Processing Capacity. This was assessed through two different tasks: a lateralised reaction time with a counting backward task, and a visual divided attention task.

In the lateralised reaction-time task, 64 stimuli appeared on the screen, 16 by each quadrant, in a non-predictable position. The visual task was the primary task. The secondary task was counting backward by steps of 3 starting at 800 . The primary task was first realised in isolation, then with the secondary task. The lowering of performance in the primary task when carried out with the concurrent task enabled us to measure the level of interference caused by the secondary task. AM's results at the primary task alone were normal: His mean reaction time was $259 \mathrm{msec}$. (controls: $315 \mathrm{msec}$, s.d.: 73). At the primary task with the counting task, AM's results remained perfectly normal: his mean reaction time was $333 \mathrm{msec}$. (controls: $395 \mathrm{msec}$, s.d.: 85).

The visual divided attention task consisted of a primary tracking task and a secondary detection task. This is a highly interfering task since it recruits very similar spatial and visual attentional processing. In the tracking task, the subject was asked to track (with the right hand) a little target rectangle that continuously changes its position on the screen along a horizontal line. In the visual detection task, the subject had to press a button (with the left index finger) when little vertical lines (displayed at irregular intervals on a horizontal line) moving at a constant pace from left to right passed through a centrally displayed window. Each task was first realised in isolation, then together. On the tracking alone, the mean distance (measured in pixels) between target rectangle and patient's rectangle was 4.27 (controls: 4.25 , s.d.: 0.85 ); in the divided condition, it was 4.48 (controls: 7.47 , s.d.: 2.7). The very good performance of AM on the divided attention task was not due to an attentional bias ward the primary task since performance on the secondary detection task was in the normal range (AM: 4.7; controls: 4.92 , s.d.: 1.17).

\section{Intellectual Efficiency}

AM's intellectual efficiency has been assessed by the Progressive Matrices and the Wechsler Adult Intelligence Scale (W.A.I.S.).

On the Progressive Matrices, AM scored 54 (95th percentile). On the W.A.I.S., AM obtained a verbal I.Q. of 110 , a performance I.Q. of 122 , W 116 AM only scored outside of the norms on the digit span subtest (scaled score: 6). These results indicate a normal intellectual efficiency.

\section{Frontal Tests}

Given the presence of frontal lesions, four tests sensitive to frontal dysfunction were administered.

A "Tower of London" Planning Task. This was elaborated and administered following the description given by Shallice (1982). There were 12 problems requiring $3,4,5$, and 6 moves, with 3 problems at each length. 12 problems requiring $3,4,5$, and 6 moves, with 3 proble AM's performance was perfectly normal: he scored $3,4,6.7$, and subjects: 3.8 , s.d. $0.8 ; 6.8$, s.d.: $2.8 ; 7.8$, s.d.: $0.7 ; 9.8$, s.d.: 0.4$)$.

A Modified Card Sorting Test (Nelson, 1976). AM's performance was strictly normal: He achieved six categories, making only one perseverative error.

A Stroop Test. The Nehemkis and Lewinshon version (1972) was administered. On the colour dots naming task, AM was a little too slow: administered. On the colour dots naming task, AM His naming time was $78 \mathrm{sec}$. (controls: $62.7 \mathrm{sec}$. , .d. black print reading task, he was a (controls: $44.3 \mathrm{sec} .$, s.d. $=9.9$ ). Finally, on the colour interference task, AM's result was almost in the normal range: His reading time was $139 \mathrm{sec}$ (controls: $105 \mathrm{sec}, \mathrm{s} . \mathrm{d} .=27$ ). The error scores for the 3 conditions were (controls: $105 \mathrm{sec} .$, s.d. $=27$. The error scores for the controls, AM never in the normal range. Furthermore, and contrary to controls, AM never excessive carefulness.

A Phonemic Fluency Test. (Letters P, F, and L.) AM scored in the normal range: $\mathrm{H}$ (sulerated a total of 35 words (controls: 29.3 , s.d.: 6.7)

Considered overall, these findings did not suggest frontal dysfunctioning. Furthermore, thi sbject's judgement was not altered; he reacted 
appropriately to everyday situations and his social adjustment was essentially normal.

\section{Long-term Memory Assessment}

We administered two verbal memory tests and one nonverbal memory test. The two verbal tests were the Auditory Verbal Learning Test (Taylor's [1959] version) and a recall and recognition task (Bertaux et al., Note 3); the nonverbal task was the "Ruche" Test (Violon \& Wijns, 1984).

On the Auditory Verbal Learning Test, AM's recall scores on each trial of the A-list were $6,13,15,15,15$. These results were within the normal range except for the first trial (Lezak, 1983). After learning the interpolated wordlist (B-list), the patient again recalled 15 words of the A-list; thus he clearly remained within the normal range. Finally, he recalled 13 words and recognised 14 words after a 30 -minute delay; these results can also be considered as normal.

In the recall and recognition task, a list of 40 related words was presented to the patient. The words fell into 8 semantic categories, 5 words per category. Words from the same category were presented successively, following their category label. Then, the patient received a cued recall test and a 2 -item forced recognition test. AM recalled 15 words and recognised 35 words. Controls recalled an average of 22.3 words (s.d.: 7.6 ) and recognised an average of 36.8 words (s.d.: 2.3). AM's results were just within the normal range at recall and normal at recognition.

The "Ruche" Test (Violon \& Wijns, 1984) consisted of the presentation (for $45 \mathrm{sec}$.) of a matrix of 41 rhombs of which 10 , located at different positions in the matrix, are coloured black. The patient was asked to memorise the position of the black rhombs in order to colour them in on an empty response matrix. The task consisted of 5 successive recall trials and 2 delayed recall and recognition trials. AM obtained the maximum score (10 rhombs) at the immediate and delayed trials.

In summary, AM presented well-preserved intellectual, language, and perceptual abilities and no frontal impairment. Finally, the examination did not reveal any deficit of long-term memory, nor any disturbance of attentional functions (even on divided attention tasks).

\section{CHARACTERISTICS OF THE WORKING MEMORY DEFICIT}

\section{Immediate Memory Spans}

AM's immediate memory span was tested with respect to different materials and both a repetition and a recognition response me $d$ were used (results can be seen in Table 1)
TABLE 1

Span Sizes on the Different Span Tasks

\begin{tabular}{lcc}
\hline & $A M$ & Controls \\
\hline Digit Span & & \\
$\quad$ Auditory presentation & 5 & $6.9(0.99)$ \\
$\quad$ Visual presentation & 4 & $7.0(1.05)$ \\
Block-tapping Test & 4 & $5.7(0.82)$ \\
Recognition Span & & \\
$\quad$ Objects & 4 & $4.5(1.01)$ \\
Abstract designs & 2 & $3.5(1.57)$ \\
Sentence Span & 0 & $3.1(0.80)$ \\
\hline
\end{tabular}

Forward digit spans were tested in auditory and visual modalities. Lists of 2 to 9 digits were presented either auditorily (read by the examiner) or visually (on cards) at the rate of 1 digit per second. Three sequences at each digit length were presented until the patient failed 2 out of the 3 ach digit length were presented until the patient failed 2 oul of the 3 sequences of the same length. His span was 5 in the auditory presentation (controls: $6.9 ;$ s.d. $=0.99$ ) and 4 in the visual one (controls: 7 ; $\mathrm{d}=1.05$ ). Visuo-spatial span was assessed by the Corsi block-tapping procedure (Milner, 1971). AM obtained a span of 4 (controls: 5.7; s.d. $=0.82$

We also administered 2 visual recognition span tasks on meaningful (objects) and meaningless (abstract designs) material (Belville, Lussier, \& Peretz, Note 1). Sequences of 2 to 8 items were presented to the patient. Peretz, Note 1). Sequences of 2 to 8 items were prest item on a given trial There were 4 trials at each list length. After the last item on a given from was presented, the patient was shown a random array of the indicate the items which the sequences were selected. Then, his task was to indicate the item just presented in the correct serial order. Testing continued until the patien failed 3 out of the 4 trials of the same length. AM obtained a span of 4 failed 3 out of the object material (controls: $4.5 ;$ s.d. $=1$ ) and a span of 2 on the abstract design material (controls: 3.5 ; s.d. $=1.57$ ).

In addition, AM was assessed for sentence span by using Daneman and Carpenter's (1983) technique. This is a test devised to tax both the processing and storage functions of working memory rather than just the storage sing and storage functions of working memory 2 to 6 sentences of 13 to 16 functions. The patient was given sequences of 2 to 6 sentences of 13 to 16 words. He was asked to read the sentences aloud and to remember the last word of each. He then had to recall as many last words as possible in any order. There were 5 trials at each length. The span corresponded to the length at which the patient succeeded in recalling the last words in 3 the length at which the patient 2 words at the following length, he received a bonus of 0.5 points. At this task, AM scored very low: following Daneman and Carpen s formula, he scored 0 (controls: 3.107 , s.d. $=0.80)$. 
All spans were below the average score of the controls except for the recognition spans with meaningful and meaningless material, which were in the normal range and just within the normal range respectively.

We have attempted to interpret this moderate span reduction in terms of Baddeley's (1986) working memory model, but as the subcomponents involved in the visuospatial sketchpad have to date been less widely explored and are less well understood, we have only evaluated the subcomponents of the slave system responsible for the retention of speechbased information, that is, the articulatory loop system.

\section{The Articulatory Rehearsal Mechanism and the} Phonological Store

To assess whether the articulatory rehearsal process and the phonological store could function normally, we administered span tasks intended to show the existence of a word length effect and of a phonological similarity effect. The word length effect and the phonological similarity effect with visual presentation have been considered to reflect the use of the articulatory rehearsal mechanism. These effects disappear if the subject is prevented from rehearsing by being asked to utter some sounds continuously (articulatory suppression). As to the phonological similarity effect with auditory presentation, it should reflect the operation of the phonological store.

\section{The Phonological Similarity Effect}

We have assessed the patient's span for phonologically similar and dissimilar items in the auditory and the visual modalities. Five sequences of two to six similar and dissimilar monosyllabic words have been prepared. None of the words was repeated in the different sequences. In the auditory condition, words were spoken by the experimenter; in the visual condition, upper-case words printed in black were presented on white cards. The item sequences appeared in ascending order and at a rate of one item per second. The patient was asked to repeat the sequence in the correct order. Presentation of further sequences continued until the patient failed to recall, in the correct serial order, three out of the five sequences at a given length. Furthermore, the effect of articulatory suppression was investigated in the visual condition. The patient was required to count repeatedly from one to ten during presentation. The same procedures were administered to ten control subjects.

$\mathrm{AM}$ and the control subjects showed the typical advantage of phonologically dissimilar words over phonologically similar words in both modalities (see Table 2). Furthermore, with visual presentation, the articulatory suppression abolished the phonological similarity effect in both $t$ and the controls.
TABLE 2

Memory Span of AM and Controls (Mean Scores and Standard Deviation) for Cols

\begin{tabular}{|c|c|c|c|c|c|c|}
\hline \multirow[b]{2}{*}{ Word Type } & \multicolumn{2}{|c|}{ Auditory } & \multicolumn{2}{|c|}{ Visual } & \multicolumn{2}{|c|}{ Suppression } \\
\hline & $A M$ & Controls & $A M$ & Controls & $A M$ & Controls \\
\hline Similar & 2 & $4.6(0.51)$ & 3 & $4.4(0.51)$ & 3 & $3.6(0.69)$ \\
\hline Dissimilar & 4 & $5.5(0.52)$ & 4 & $5.4(0.51)$ & 2 & $3.3(0.67)$ \\
\hline Short & 3 & $4.8(0.42)$ & 4 & $4.8(0.42)$ & 2 & $3.8(0.63)$ \\
\hline Long & 3 & $4.2(0.63)$ & 3 & $4.3(0.48)$ & 2 & $3.7(0.48)$ \\
\hline
\end{tabular}

\section{The Word Length Effect}

The procedure we have adopted is similar to that for the phonological similarity effect except that the item sequences were composed of short similarity effect except that the ithoble) words. Furthermore, there were (1-syllable) and long (3- or 4-syllable) words. Furthermore, there wer to high-frequency short and long words (frequency of occurence count). Both the patient 15 in the Vikis-Freiberg (1974) word frequency in span performance with long and the control subjects showed a decrease in span performance with long vords compared to short words in the visual condition (see Table 2 ). With auditory presentation, AM's performance on the short-word span was comparable to that on the long-word span. Yet, if we compute the total pro列 3 portion of items recalled in the approprate serial orte advantage of short sequences of the lengths 2,3 , and 4 , wort words against $84 \%$ for the long vords over long words ( $88 \%$ for the short words against $8 \%$ wisual presentation words). In any case, the articulatory suppression with visual presentation produced a reduction of the span and abolished the word-length effect in both $\mathrm{AM}$ and the controls.

In summary, AM showed the expected phonological similarity and word length effects on both auditory and visual presentation, as well as the length effects on both audito articulatory suppression on visual presenabolition of these effects with the articulationt was able to use the articulatory tation. These results suggest that the patient was able to use the articulatory rehearsal mechanism and phonological store. As these components of

working memory did not seem to be involved in AM'
it remained to explore central executive functioning.

\section{The Central Executive}

In order to assess the central executive system, we have adapted the verbal and nonverbal procedures used respectively by Morris (1986) and Sullivan, and nonver on A.D. patients. They Corkin, and Growdon (1986) in studies conducted on A.
consisted of Brown-Petr on tasks (Brown, 1958; Peterson \& Peterson, 
1959) in which the subject had to retain infra-span verbal or nonverbal material over different short delays filled by distractor tasks varying in complexity.

\section{The Verbal Task}

Vallar and Baddeley (1982) showed that articulatory suppression leads to minimal forgetting in the Brown-Peterson task with three visually presented consonants, whereas the performance is severely disrupted by counting backward. They concluded that the retention of the consonant sequences relies on maintenance rehearsal, a process that is achieved by the central executive without the support of the articulatory loop system. According to Morris (1986; see also Morris \& Baddeley, 1988), if the central executive is defective in AM (if its capacity is reduced), it will be unable to cope with the processing demands of the maintenance rehearsal processes and with the demands of the distractor task. Moreover, the deficit will be greater as the distractor tasks become more complex. In other words, if the tasks require a greater part of the processing resources of the central executive, the latter would be unavailable to regulate the maintenance rehearsal.

The verbal task was a closed adaptation of the Morris (1986) paradigm. The patient had to retain 3 verbal items (consonant trigrams) and to recall them after delays of $0,5,10$, and $20 \mathrm{sec}$. The trigrams were presented on cards and were selected to avoid acoustic confusability and known abbreviations. There were 4 conditions: unfilled intervals and intervals filled with either articulation, digit reversal, or digit addition. Articulation requires few central processing resources (Baddeley \& Hitch, 1974) and causes little forgetting on the Brown-Peterson task in young subjects (Vallar \& Baddeley, 1982), contrary to digit reversal and especially digit addition, both of which are more demanding and give rise to more forgetting (Dillon \& Reid, 1969). We administered 6 trials for each delay within each condition. In the unfilled interval condition, the patient was asked to concentrate on remembering the trigrams but not to rehearse them vocally. In the articulation filled condition, the patient had to count repeatedly from 1 to 10 . In the digit reversal and digit addition conditions, the patient was instructed to reverse or add series of 2-digit numbers. Performance was measured in terms of the mean percentage of consonants that were recalled in the correct position (see Fig. 1).

With no distraction, AM showed virtually no forgetting. With articulation, the difference between AM and the controls was minimal but AM's deficit increased dramatically with digit reversal and digit addition: For all delay intervals, he recalled $98.6 \%$ of the items in the unfilled condition (controls: $100 \%$ ), $91.7 \%$ in the articulation condition (contrc ${ }^{\circ} \cdot 99.1 \%$,
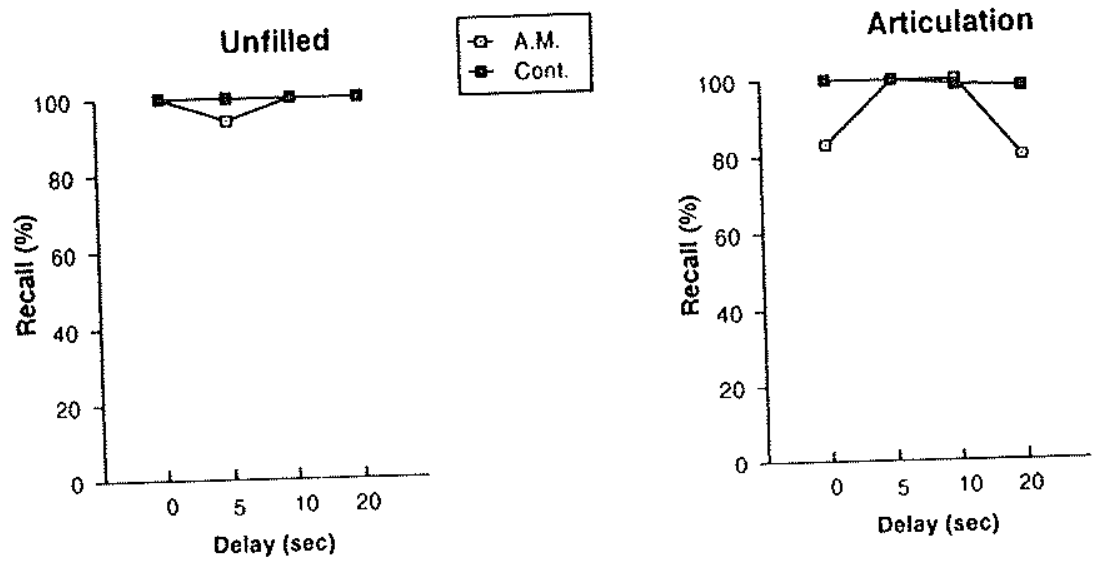

Reversal
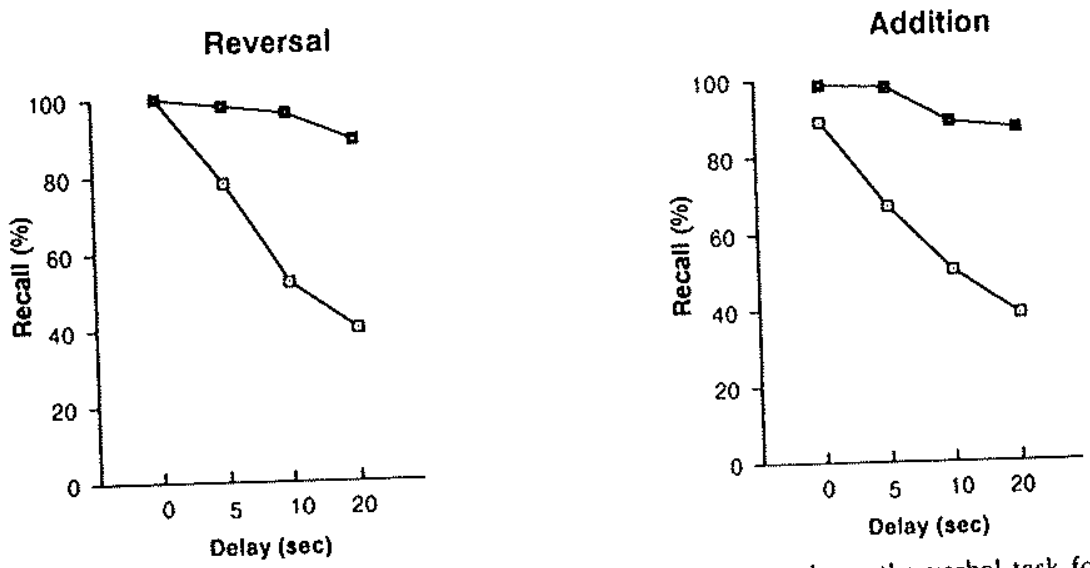

FIG 1 Percentage of controls on the verbat task for each delay with unfilled and filled intervals.

s.d. $=3.27$ ), $68 \%$ in the reversal condition (controls: 95.8 , s.d. $=7.76$ ), and $61.1 \%$ in the addition condition (controls: 93.2 , s.d. $=10.1$ ). Moreand $61.1 \%$ in the controls became more and more important as the delay increased.

\section{The Nonverbal Task}

In a study exploring the relationship between the visuospatial sketchpad and the central executive system, Morris (1987) suggested that the central and the central executive system, Morsibly in the executive is principally involved in the encoding (and possiblenance retrieval) of visuospatial material and that its role in the maintenance rehearsal of such material is quite minor. He has shown how an interferent
activity (tracking) introruced during a $10-\mathrm{sec}$. delay between presentation 
and recall did not disrupt performance of normal subjects on a spatial memory task requiring subjects to recall the locations of circles randomly presented in a square. On the other hand, the tracking task interfered with visuo-spatial processing when this was performed during stimulus presentation.

The nonverbal task was adapted from that reported by Sullivan et al. (1986). It incorporated distractor-free and distractor-filled retention intervals. Recall was tested after delays of $0,5,10$, and $20 \mathrm{sec}$. Six trials were given at the 4 retention intervals for each condition. The examiner touched 2 of 9 blocks that were displayed irregularly on the board that is usually used for the Block-Tapping Test (Corsi, 1972). At the end of the interval, the patient was asked to touch them in the same order. During the distractor-filled retention intervals, the examiner touched a point on one of the subject's hands and the subject touched that point on the other hand. The performance was scored in terms of the mean percentage of blocks recalled in the correct location. Globally, AM recalled $87.5 \%$ of the blocks in the unfilled condition (controls: 99.4, s.d. $=1.35$ ) and $62.5 \%$ in the filled condition (controls: 91.8, s.d. $=7.83$ ). These findings indicate that the patient and the control group displayed poorer recall following filled rather than unfilled intervals. However, the extent of forgetting on the unfilled condition was greater for AM; moreover, AM was more severely disrupted by the distractor task than were the control subjects. Figure 2 shows the results for each delay interval.

An important deficit in AM's performance was present in the filled condition for the $5 \mathrm{sec}$. delay interval and remained unchanged for the longer intervals. AM also showed some difficulty in the unfilled condition for the $10 \mathrm{sec}$. intervals. In fact, he showed more forgetting for nonverbal material than for verbal material.
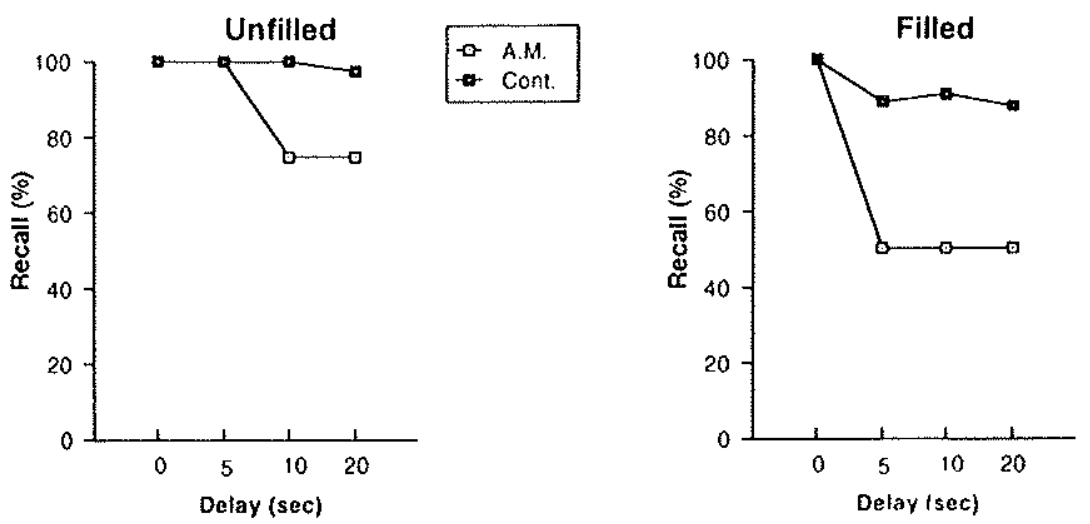

FIG. 2 Percentage of blocks recalled by AM and the controls on the nonvert "ask for each delay with unfilled and filled intervals.
In summary, AM showed significant forgetting on both verbal and nonverbal Brown-Peterson tasks when distraction was present. Concerning the verbal task, the quasi-normal performance of $A M$ in the unfilled interval condition indicates that the maintenance rehearsal mechanism functioned normally. Nevertheless, it could be argued that there was a disruption of the maintenance rehearsal mechanism so that consequently, in remembering the consonant trigrams, the patient was forced to rely on the articulatory loop system. It would follow, then, that as the effect of articulation is to disrupt the operation of the articulatory loop, we should have observed a significant forgetting in the articulation condition, yet this was clearly net the case. We must thus conclude that maintenance rehearsal was intact. The fact that AM's deficit increased with the complexity of the interferent task and with delay supports the hypothesis that his working memory deficit was caused by a reduction in the capacity of the central executive system. More specifically, the resources of the patient were insufficient to meet the demands of the distractor tasks and maintenance rehearsal simultaneously. In completing the nonverbal task, AM showed some forgetting in the absence of an interfering task but his deficit was much more important when distraction was present. From the perspective of Morris's (1987) when distraction was present. From the perspective of Morris's (1987) could be different from the mechanism that contributed to forgetting for could be different the nonverbal task verbal material: The reduction of AM's recall in the nonverbal task could
be the consequence of an abnormal interference between the memory and the distractor task material. The decrement of AM's central capacity should lead to faulty encoding and to less noticeable traces, which could explain poorer recall in both unfilled and filled conditions. In line with Morris's (1986) suggestion concerning the deficit of Alzheimer patients, Morris's (1986) suggestion concerning the deficit of Alzheime of AM's deficits in the verbal task.

The next experiment was designed to address separately the processing and storage aspects of the central executive. With this experiment, we wanted to consider a question which is at the heart of Baddeley's view: If there exists a single resource pool for different tasks, when a patient has fewer resources at his disposal and has to share these resources between storage and processing, will these two aspects of the central executive be penalised?

\section{Assessment of the Processing and Memory Components of the Central Executive}

AM was assessed on a verbal span test devised to allow us to assess the impact of the reduction of central executive resources on processing and storage. This task $w_{a}$ nodelled after that conceived by Daneman and 
Tardif (1987) and it was presented to AM in January 1990 (15 months after the main neuropsychological examination). During these 15 months, AM's working memory had gradually improved yet remained significantly impaired (especially on dual tasks). Table 3 shows the results of the control testing performed during the last 6 months of 1989 .

In the verbal span test, the patient was presented with cards containing a series of 4 separate words such as "MUSE," "AU," "VENT," "BERGE." On each card, new words could be created without changing the original order of the given words (in the above example, they are MUSEAU, AUVENT, and AUBERGE). For some words (AUVENT and AUBERGE), the junction-point between the 2 original words is situated at a syllable boundary (AU-VENT, AU-BERGE). For the other word (MUSEAU), there is no syllable boundary at the junction point (MUSE-AU). The patient's task was to find as quickly and accurately as possible the new word that did not contain a syllable boundary between the 2 given words. The cards were presented 1 at a time in sequences of 2,3 , and 4 cards (there were 3 items at each sequence size, thus 27 cards in all). The test comprised 2 conditions (27 examples in each condition): a memory-loaded condition and a memory-free condition. In the memoryloaded version, the patient was asked to recall the correct words at the end of each sequence $(2,3$, or 4 words) in any order. In the memory-free version, he did not have to recall the responses. The patient received the memory-loaded condition before the memory-free one. Three measures were computed (Table 4 presents the mean performance of AM and the 10 control subjects for each measure):

1. A memory-loaded measure of the processes: The total number of correct words provided by the patient.

TABLE 3

Performance of $\mathrm{AM}$ on the Control Examination

\begin{tabular}{lcc}
\hline & AM & Controls \\
\hline Digit Span & & \\
$\quad$ Auttitory presentittion & 5 & $6 . \mathcal{K}(0.94)$ \\
Block-tapping Test & 6 & $5.76(0.82)$ \\
Reading Span & 2 & $3.12(0.71)$ \\
Verbal Brown-Peterson Task & & \\
$\quad$ Filled interval (addition, 20sec.) & 3.3 & $86.60(10.86)$ \\
Nonverbal Brown-Peterson Task & & \\
$\quad$ Filled interval (20sec.) & 50 & $87.60(13.67)$ \\
\hline
\end{tabular}

TABLE 4

Mean Performance of AM and the Control Subjects on the Verba Span Task

\begin{tabular}{lllll}
\hline & \multicolumn{3}{c}{ Controls } \\
\cline { 4 - 5 } & AM & Mcan & S.D. \\
\hline Memory-loaded Measures $(\max =27)$ & & & \\
$\quad$ Processes & 26 & 25.00 & 2.60 \\
$\quad$ Memory & 17 & 22.10 & 2.96 \\
Memory-free Processes $(\max =27)$ & 23 & 24.60 & 1.95 \\
\hline
\end{tabular}

2. A measure of the memory for the products of the processes: The 2. A measumer of correct words recalled at the end of each sequen.

A total number 3. A memory-free measure of the vertient in the condition where he did not have to store the responses.

AM showed normal scores on the process measures. Moreover, the AM showed nomal equivalent to processing score obtained in the memory-free condition. Thus, the processing effithat obtained in the me storage requirements of the task. On the ciency was not affected by the storage requirements of the task. On other hand, AM's scores on the memory measure were below the nor range. Moreover, if we consider the 5 controls (out $A M$ that is, a score memory-loaded processing score equivalent to that of $A M$, th of 26 and 27, we observe that these subjects recalled a mean of 24.4 $(\mathrm{s} . \mathrm{d} .=2.3$ ) correct responses whereas AM recalled only 17 words. Finally, s.d. $=2.3$ ) correct responses whe at the the end of the 3 - and 4 -card sets, 2 -card sets and only $67 \%$ and $45 \%$ at $100 \%, 87 \%$, and $83 \%$ of the whereas the equivalent

responses respectively.

In conclusion, the evidence from this verbal span task suggests that the central executive dysfunction observed in AM was strictly a storage capacity deficit: AM disposed of fewer total resources, and on a task that required both the processing and storage of the processing products, th

resources were allocated to processing to the detriment of storage.

In the last experiment (conducted in September 1988), we wanted to explore the consequences of such a deficit on primacy and recency effects in an immediate free recall task, that 


\section{Primacy and Recency Effects}

In order to determine whether AM would show primacy and recency effects, he was tested by being presented with a total of 18 lists of 122 - or 3 -syllable words at a rate of $2 \mathrm{sec}$. per word (frequency of word occurrence was superior to 1 in the Vikis-Freibergs, 1974, word frequency count). There were 2 conditions: free recall ( 12 lists were administered, 6 auditorily and 6 visually) and recall from end ( 6 lists were presented, 3 auditorily and 3 visually). In the free recall condition, the patient was instructed to recall as many words as he could in any order he wished. In the recall from end, he was asked to begin by recalling as many words as possible from the end of the list and then to recall as many other words as possible

Figure 3 shows the probability of recall as a function of serial position in $\mathrm{AM}$ and control subjects on the free recall and recall from end condi-

\section{Free recall}
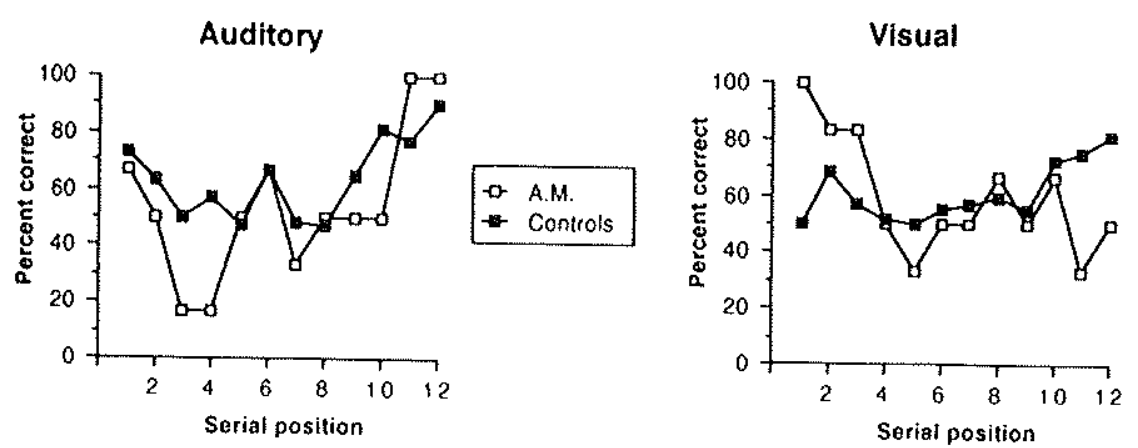

Recall from end
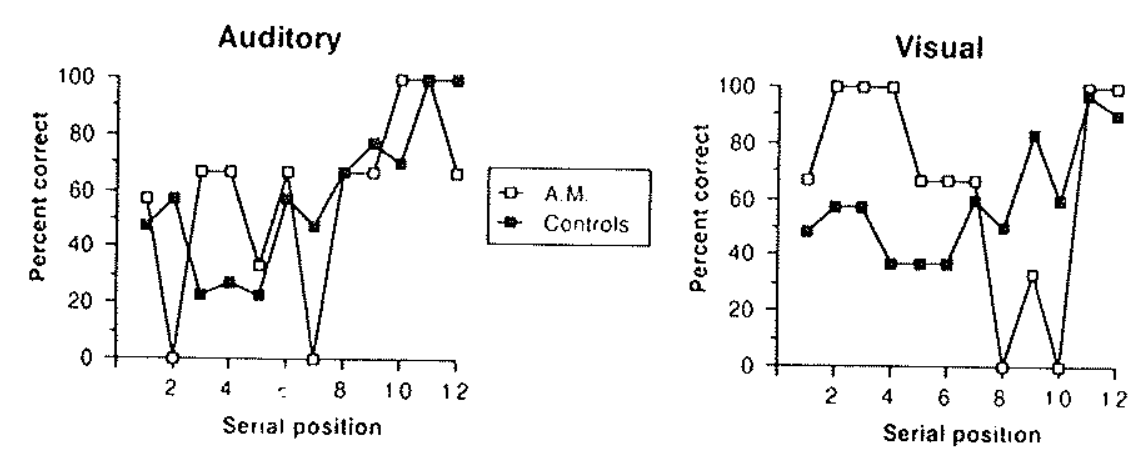

FIG. 3 Free recall and recull from end: Auditory and visual presentation $M$ and ten control subjects). tions. On the free recall condition, AM's pattern of performance was completely different with visual and auditory presentation. In the auditory modity AM showed primacy and recency effects. In the visual modality, modality, AM shory pronounced primacy effect with no recency. On the recall he showed a very pronounced primacy effect with no recency effect of AM was from end condition, with auditory modality, the recency effect of AM was strengthened, and with visual modality, a recency effect appeared but was strictly limited to the two remained very important.

In addition to the serial position curve, we measured recency by means In additon of the Tulving and Colotla (1970) measure. It assigns to primary meen their words that are recalled with seven or fewer items intervening between their presentation and their retrieval. Words with an intertral Table 5 presents are considered to be recalled from secondary memory. Table controls. the primary and secondary memory measures of AM and of ten controls.

With auditory presentation, the primary memory measure is in the normal range whereas it is below the normal range with visual presentation both in the free recall and recall from end conditions. The secondary both in the free recall and recall from end con memory processes. The memory scores are assumed to reflect long-term that of the controls.

Finally, we have examined AM's order of recall by means of Postman and Phillips' (1965) recency score. This score was based on the number of

\section{TABLE 5}

Primery and Secondary Memory Scores of AM and Ten Control Subjects

\begin{tabular}{llll}
\hline & & \multicolumn{2}{c}{ Controls } \\
& AM & Mean & S.D. \\
\hline Primary Memory & & & \\
Free recall (6 lists) & & & \\
$\quad$ Auditive presentation & 3.3 & 3.1 & 0.91 \\
$\quad$ Visuat presentation & 1.3 & 2.6 & 1.03 \\
Recall from end (3lists) & & & \\
$\quad$ Auditive presentation & 4.0 & 3.4 & 0.61 \\
$\quad$ Visual presentation & 2.3 & 3.3 & 0.66 \\
Secondary Memory & & & \\
Free recall & & & \\
$\quad$ Auditive presentation & 3.2 & 4.6 & 1.94 \\
$\quad$ Visual presentation & 5.7 & 5.3 & 2.00 \\
Recall from end & & & \\
$\quad$ Auditive presentation & 2.7 & 3.8 & 1.67 \\
$\quad$ Visual presentation & 5.6 & 4.0 & 2.15 \\
\hline
\end{tabular}


times the first word recalled came from the second half of the list. In the free recall condition, there were 6 lists and, consequently, the recency score could vary from 0 to 6 . AM's recency score was 6 with auditory input (controls: $4.4 ;$ s.d. $=1.5$ ) and 0 with visual modality (controls: 5.2 ; s.d. $=1.03)$. Thus, in the visual modality, the first item recalled by AM never came from the second half of the lists: As a matter of fact, AM began with words that were located at the first 3 positions of the list for 5 out of the 6 trials. In the recall from end condition with auditory and visual input, all the first items came from the second half of the lists both in AM and the control subjects.

To summarise, in free recall and for visually presented words, AM showed a lack of recency effect, an important primacy effect, and he recalled the initial words of the lists first. In the auditory modality, he presented a recency effect and he recalled the last words of the lists first. When he was asked to recall from the end, he was able to follow the instructions and presented a limited recency effect for visually presented words. Finally, his primary memory capacity was clearly defective with visual input whereas his secondary memory capacity was normal.

Following Vallar and Papagno (1986), the recency effect in immediate free recall of auditorily presented words reflects the application of an ordinal retrieval strategy to the output of the phonological store. Concerning auditorily presented items, their storage in the phonological store is considered as relatively automatic whereas visual items must undergo a phonological recoding and be conveyed to the phonological store by means of the articulatory rehearsal. As Vallar and Papagno (1986) point out, this association between the recency effect in free recall of auditory material and "automatic storage" in the phonological store is illustrated by studies showing that concurrent tasks affect recency in immediate free recall for visually but not auditorily presented material (Anderson \& Craik, 1974; Bartz \& Salehi, 1970; Silverstein \& Glanzer, 1971). The fact that the recency effect is not disrupted by a concurrent task in the free recall of auditory items suggests that, in this particular case, it is not very dependent upon central executive resources

Such a view implies an interpretation of AM's performance pattern. The reduction of AM's processing resources still allowed him both to initiate long-term memory processes (see the primacy effect) and to apply a recency strategy to the output of the phonological store. On the other hand, in the free recall and visual input conditions, owing to the reduction of available processing resources, the patient did not initiate the recoding and conveying operations of visually presented items (operations which are demanding for the central executive). Therefore, only the initial longterm memory operations were executed spontaneously (see the robust primacy effect, the output order in which beginning it $s$ were pre- dominant, and the lack of recency effect). As a matter of fact, the patient seemed to give precedence to long-term memory processes. If he was asked to recall last words first, we observed that with auditory input, the recency effect was strengthened and the recall from other positions was reduced (see the reduction of the secondary memory capacity). With visually presented items, a recency effect appeared but was very limited (see the sented items, a recency effect appeared but was very limited (see the primary memory capacity). This suggested that the patient was able to recode visually presented material and to use a recall from end strategy. However, as the recoding of visual information necds processing capacity, he could devote fewer resources to storage; thus the reduction of the recency effect is explained. It may also be possible that the recency effect we observed in the recall from end condition for visual items reflected the we observed a visual short-term store (Warrington \& Shallice, 1972). If this interpretation is correct, we must assume that the "visual" coding of visually presented items also required some of AM's processing resources, provoking a reduction of his storage capacity.

\section{GENERAL DISCUSSION}

In this study, we have described the case of a head-injured patient whose intellectual and cognitive skills, long-term verbal and nonverbal memory performance, and attentional abilities were entirely satisfactory but who showed a reduction in memory span for verbal and nonverbal material.

The short-term memory deficit was investigated with regard to Baddeley's (1986) working memory model. Following this model, a reduction in verbal and nonverbal spans could be imputed to two distinct distion in verbal and nonverbal spans could be imputed to two distinct disorders affecting the central executive, or both. Several characteristics of AM's performance seem to suggest that his verbal span deficit cannot be attributed to a dysfunctioning of the articulatory loop system. Indeed, the observation in AM of word length and phonological similarity effects, as well as the abolition with articulatory suppression of the visual and auditory word length effects and of the visual phonological similarity effect, appears to indicate that both the phonological store and the articulatory rehearsal mechanism were functioning. As a matter of fact, the deficit AM showed on the verbal Brown-Peterson task when the distractor tasks were more demanding suggests a reduction of the central execcutive resources. This resource reduction prevented the patient both from accomplishing the distractor tasks and from co-ordinating the processes involved in maintenance rehearsal. Our investigations mainly assessed AM's performance on verbal material. As for the visuospatial span reduction, the data we $h$ - collected does not allow us totally to rule out the 
participation of the visuospatial sketchpad in the deficit. However, the fact that the patient was clearly impaired on the Brown-Peterson tasks, both with verbal and nonverbal material, is consistent with the hypothesis that the central exccutive system was involved in both the verbal and spatial span reduction. Evidence from Morris' (1987) study leads us to suggest that AM's central executive deficit should have a greater effect on the encoding of visuospatial material (with the consequence of stronger interference effects) than the maintenance rehearsal.

It remains for us to account for the way in which this reduction of central executive resources caused the decrement of AM's spans. The verbal memory span is considered to be dependent on both the articulatory loop system and the central executive (Baddeley, 1976; Vallar \& Papagno, 1986). The articulatory loop system is able to store in the correct order only a limited number of items but the central executive may increase this number either by improving the working of the articulatory loop (for example, by grouping the items in higher level units) or even by storing information itself. Following Baddeley (1986), the central executive is also involved in the retrieval process and in the interpretation of the information retrieved from the articulatory loop system. In this regard, an impaired span is consistent with a reduction of central executive resources and with a normal functioning of the articulatory loop system. AM's central executive probably does not allocate sufficient resources to co-ordinate the activities of the slave systems and its own operations. The relatively better performance of AM on the recognition (pointing) span tasks could be related to the fact that the retrieval demands on the central executive are less important in a recognition span than in a standard span task.

We have also shown that the decrement of AM's central executive resources have modified the strategies he used in an immediate free recall task on visually presented word lists: More precisely, he did not spontaneously initiate all the operations involved in the recency strategy (especially the subvocal recoding of visual material, which must be a demanding operation) and he favoured long-term processes.

Our investigations thus suggest that by AM's resource reduction, different processes involved in short-term storage were indirectly affected: In some tasks, this compromised maintenance rehearsal, caused faulty encoding and probably disturbed retrieval of information from the slave systems; in other tasks, it prevented the normal execution of the slave systems operations.

Other aspects of the study yield a more precise characterisation of AM's central executive deficit and two additional conclusions may be proposed.

First, AM's deficit seems to be restricted to one aspect of the central executive; the processing resources. When sufficient resources were available, the control, planning, and storage processes that are the responsibility of the central executive seemed to operate normally. There is abundant evidence for the preservation of the control and planning processes: no impairment of overall cognitive abilities, absence of frontal syndrome, and normal processing results on the memory-free condition in the verbal span task (Daneman \& Tardif, 1987). Concerning the storage processes, provided the central executive was not engaged in another demanding cognitive task, maintenance rehearsal was intact (see near-normal performance on the verbal Brown-Peterson task with both unfilled interval and articulation).

Second, AM's resources are not generally reduced and his deficit can be narrowly referred to as a "storage capacity" deficit (Foos, 1989). The patient obtained normal results on both divided attention tasks (reaction time with counting and tracking with detection), which should have been time with counting and tracking with detection), which should have decit, then, appeared on divided attention tasks only when one task required storage of information. Furthermore, when the patient had to share his diminished resources tion. Furthermore, when the patient had to share his diminished resources between processing and storage of the products of priment of storage. This is processing functions were prioritised to the detrion in Daneman and Tardif's task.

Indeed, AM's processing seemed only to be affected when the storage of substantial information was absolutely necessary for its execution and when processing was demanding. As such, AM complained about difficulwhen processing was demanderstanding scientific papers, in attending to and ties in reading and understanding scientific papers, in attending to and understanding technical conversations, and in solving auditorily arithmet-
ical problems, because he was unable to maintain information in "active state" during the execution of mental operations. If we interpret AM's discourse processing difficulties with respect to the elaborated capacity discourse processing dificulties washer (1988), the storage capacity deficit model proposed by Zacks and Hasher (1988), the storage capacity deficit can be assumed to have prevented AM from retaining in working memory information derived either from preceding text or from prior knowledge retrieved from long-term memory. As the relevant information was no available in working memory, the processing operations (such as inference generation) could not be executed and, consequently, comprehension was disrupted. However, AM's deficit in text processing occurred particularly for complex scientific or technical material: Comprehension of such for complex scientific or techial required many processing resources, thus rendering AM's remaining resources insufficient to store already processed information.

In conclusion, AM did not show evidence for a structural deficit of the In conclusion, AM did not similarly, the system's storage functions seemed to be intact. The different investigations system's storage functions seemed central executive disposed of fewer processing resources. suggest that AM's central executive dunctions of the central executive were essentially 
affected by AM's resource reduction. In other words, the patient seemed to allocate his limited resources to processing at the expense of storage. Such a differential resources allocation could be the result of a strategic adaptation on the part of the patient. It could also suggest a fractionation of processing resources, some being devoted to storage and others to ongoing processing. However this interpretation, postulating the existence of different resource pools, is incompatible with AM's performance on the verbal Brown-Peterson task. Indeed, the increase of AM's deficit in recall, as the complexity of the interfering task and the delay increased, more plausibly supports the notion of a reduction in a general resource pool and of a strategic adaptation of the patient to the requirements of the tasks. Yet such an interpretation poses another difficulty: How is it possible to explain the absence of deficit on those divided attention tasks which did not require storage? It could be that the demands of these divided attention tasks were below the limited resources still available to AM. In any case, this differential sensitivity of the patient to interference in dual tasks with and without a storage component requires further analysis and suggests that a more precise specification of the resource allocation in dual tasks is needed, specifically to fractionate processing resources and storage capacity.

Manuscript received 31 July 1990 Revised manuscript received 5 November 1991

\section{REFERENCES}

Anderson. C.B.M.. \& Cratk, F.I.M. (1974). The effect of a concurrent task on recall from primary memory. Journal of Verbat Learning and Verbal Behaviour, 13, 107-113.

Atkinson, R.C.. \& Shiffrin. R.M. (1968). Human memory: A proposed system and its control processes. In K.W. Spence \& J.T. Spence (Eds.). The psychology of learning and motivation: Advances in research and theory, Vol. 2. New York: Academic Press.

Baddeley, A.D. (1976). The psychology of memory. New York: Basic Books.

Baddeley, A.D. (1986). Working memory. Oxford: Oxford University Press.

Baddeley, A.D., \& Hitch, G. (1974). Working memory. In G.H. Bower (Ed.), the psych. ology of learning and motivation, Vol. VIII. New York: Academic Press.

Baddeley, A.D.. Logie, R.H., Bressi, S., Della Sala, S., \& Spinnler, H. (1986). Dementia and working memory. Quarterly Journal of Experimental Psychology, 38A, 603-618.

Bartz, W.H., \& Salehi, M. (1970). Interference in short- and tong term memory. Journal of Experimental Psychology, 84, 380-382. Brown, J. (1958). Some tests of the decay theory of immediate memory. Quarterly Journal
of Experimental Psychology, 10, 12-21.

Bruyer, R., \& Tuyumbu. B. (1981). Le test de fluence verbale dans une population belge d'expression françatise. Revute de Psychologie' Appliquée, 31, 1-11

Buschke, H. \& Fuld, P.A. (1974). Evaluating storage, retention, and retrieval in disordered memory and learning. Neurology, 24, 1019-1020.

Daneman, M., \& Carpenter, P.A. (1983). Individual differences in working memory and reading. Journal of Verbal Learning and Verbal Behaviour, 19,450-466.
Daneman M. \& Tardif, T. (1987). Working memory and reading skill re-examined. In M. Coltheart (Ed.). Attention and performance VII. Hillsdale, N.J.: Lawrence Erlbaum Associates Inc.

Dillon, R.F., \& Reid, L.S. (1969). Short-term memory as a function of information proces sing during the retention interval. Journal of Experimental Psychology, 8I, 261-269.

Foos, P.W. (1989). Adult age differences in working menory. Psychology and Aging, 4, 269-275.

Light, L.L., \& Anderson, P.A. (1985). Working-memory capacity, age, and memory for discourse. Journal of Gerontology, 40, 737-747.

Milner, B. (1971). Interhemispheric differences in the localisation of psychological processes in man. British Medical Bulletin, 27, 272-277.

Morris, G.M. Gick, M.L., \& Craik, F.I.M. (1988). Processing resources and age differences in working memory. Memory and Cognition, 16, 362-366.

Morris, N. (1987). Exploring the visuo-spatial scratch pad. The Quarterly Journal of Experimental Psychology, 39A, 409-430.

Morris, N., \& Jones, D.M. (1990). Memory updating in working memory: The role of the central executive. British Journal of Psychology, $81,111-121$

Morris. R.G. (1986). Short-term forgetting in senile dementia of the Alzheimer's type Cognitive Neuropsychology, 3, 77-97.

Morris, R.G.. \& Baddeley, A.D. (1988). Primary and working memory functioning in Alzheimer-type dementia. Journal of Clinical and Experimental Neuropsychology, 10 , Alzheimer.

Nehemkis, A.M., \& Lewinshon, P.M. (1972). Effects of left and right hemisphere lesions on the naming process. Perpetual and Motor Skills, 35, 787-798.

Nelson, HE (1976). A modified card sorting task sensitive to frontal tobe defects. Neuro psychologia, 12, 313-324.

Norman behaviour. In R.J. Davidson, G.E. Schwarts, \& D. Shapiro (Eds.), Consciousness and self-regulation. Advances in research and theory, Vol. 4. New York: Plenum Press.

Peterson, L.R.. \& Peterson, M.J. (1959). Short-term retention of individual verbal items. Journal of Experimental Psychology, 58, 193-198.

Posner M.1. \& Boics, S. (1971). Components of attention. Psychological Review, 5, 391408.

Postman, L.. \& Phillips, L.W. (1965). Short-term temporal changes in free recall. Quarterly Journal of Experimental Psychology, 17, 132-138.

Rey, A. (1959). Test de Copie d'une Figure Complexe. Paris: Edition du Centre de PsychoIogie Appliquée.

Shallice, T (1982) Specific impairments of planning. Philesophical Transactions of the Royal Society of London B, 298, 199-2019.

Shatlice, T. (1988). From neuropsychology to mental structure. Cambridge: Cambridge University Press.

Silverstein, C., \& Glanzer, M. (1971). Concurrent task in free recall: Differential aspects of L.T.S. and S.T.S. Psychonomic Science, 22, 367-368.

Sullivan, E.V., Corkin, S., \& Growdon, J.H. (1986). Verbal and nonverbal short-term memory in patients with Alzheimer's disease and in healthy elderly subjects. Develop mental Neuropsychology, 24, 387-400.

Taylor, EM (1959). Psychological appraisal of children with cerebral defects. Cambridge, Mass.: Harvard University Press.

Tulving, E., \& Colotla, V. (1970). Free recall of trilingual tests. Cognitive Psychology, I, $86-98$.

Vallar, G., \& Baddeley, A.D. (1982). Short-term forgetting and the articulatory loop. Quar. terly Journal of Experimental Psychology, 34, 53-60. 


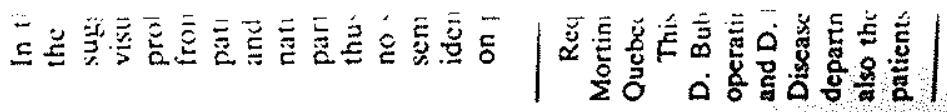

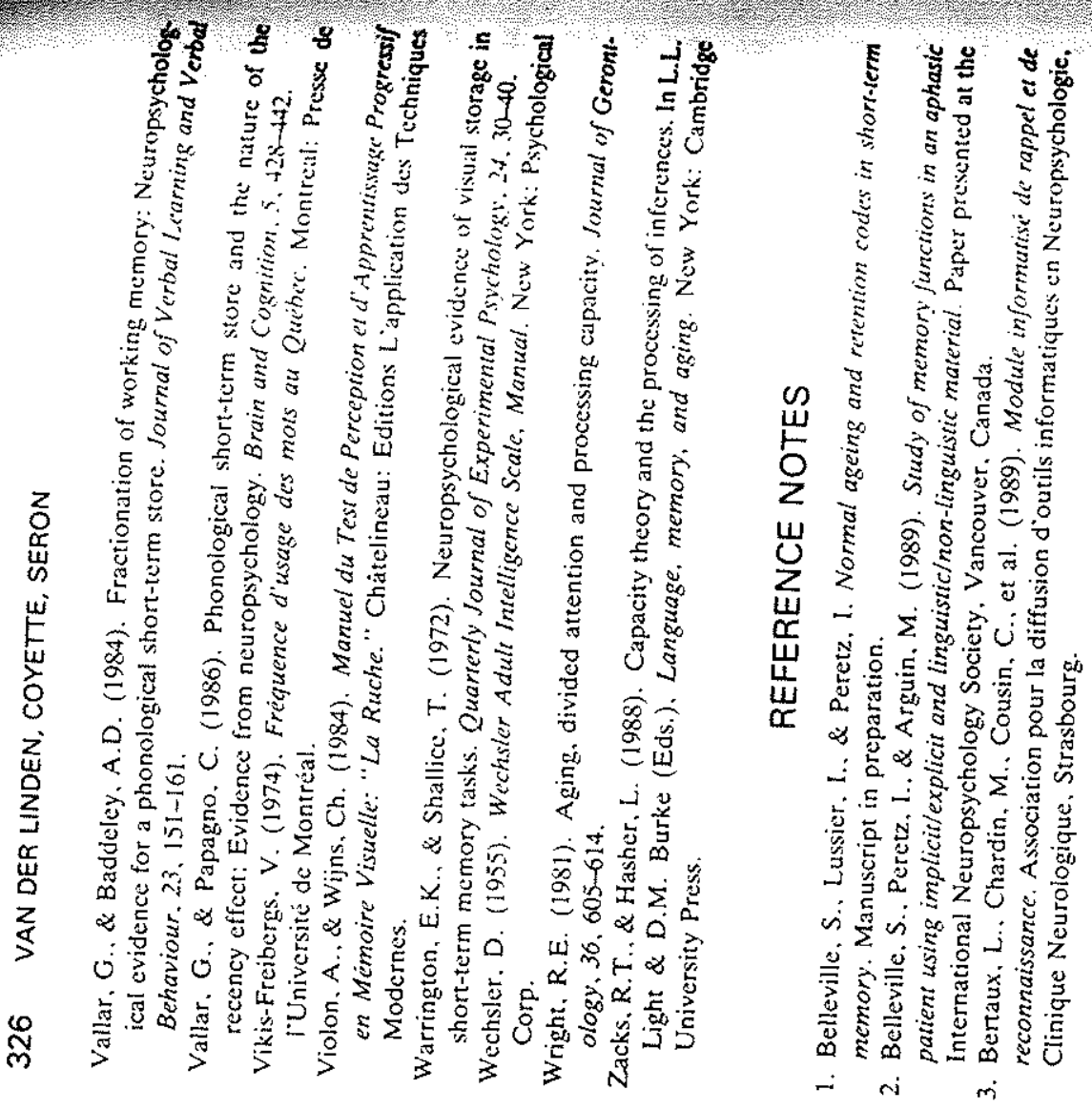

